\title{
Endoscopic ultrasound-guided creation of an enterocolostomy to relieve malignant bowel obstruction
}

\section{다)(우 $\odot$}

Authors

Adil Mir ${ }^{1}$, Parth J. Parekh², Mohammad Shakhatreh ${ }^{2}$, Paul Yeaton ${ }^{3}$

Institutions

1 Carilion Clinic - Internal Medicine, Roanoke, Virginia, United States

2 Carilion Clinic - Gastroenterology, Roanoke, Virginia, United States

3 Virginia Tech Carilion School of Medicine, Gastroenterology, Roanoke, Virginia, United States

submitted 7.11.2018

accepted after revision 19.11.2018

Bibliography

DOI https://doi.org/10.1055/a-0812-4328 |

Endoscopy International Open 2019; 07: E1034-E1037

(c) Georg Thieme Verlag KG Stuttgart · New York eISSN 2196-9736
Corresponding author

Adil S. Mir, MD, Carilion Clinic - Internal Medicine, 3386

Glade Creek Boulevard NE, Apartment 09 VA, Roanoke, virginia

Fax: +1-540-521-9018

Dr.adilshamim@gmail.com

\section{ABSTRACT}

Background and study aim Malignant bowel obstruction can cause significantly distressing symptoms in patients with end stage cancers, often requiring invasive surgical intervention. With such procedures, risks often outweigh the benefit. We report a novel application of the AXIOS stent and electrocautery-enhanced delivery system to create an enterocolostomy to bypass a small bowel obstruction in the setting of recurrent malignant bowel obstruction. A 72-year-old patient presented with recurrent malignant bowel obstruction and was deemed unfit for surgery. Endoscopic ultrasound was used to deploy an AXIOS stent, creating an enterocolostomy resolving the obstruction.

\section{Introduction}

Malignant intestinal obstruction often results in distressing symptoms and may complicate end-of-life decision-making. Surgery is invasive and the clinical setting is typically complex, introducing additional adverse events (AEs). Recovery from surgery may be prolonged, especially in patients with advanced cancers, complicated medical issues, and diminished life expectancy. With terminal cancers, the expected survival is limited and going through invasive surgical procedures increases morbidity, involving up to two-thirds of the patient's remaining life [1]. Mortality can be as high as $32 \%$ and the chances for serious AE are significant (up to $44 \%$ ) [1].

We report a novel application of the AXIOS stent and electrocautery-enhanced delivery system (Boston Scientific, Marlborough, Massachusetts, United States) to create an enterocolostomy to bypass a small bowel obstruction in the setting of recurrent malignant bowel obstruction in a patient with terminal cancer.

\section{Case report}

A 72-year-old female with past medical history of non-Hodgkin lymphoma, breast cancer, advanced metastatic pancreatic adenocarcinoma on chemotherapy, and recent history of large bowel obstruction status post-placement of a transverse colonic stent, presented with chief complaints of abdominal pain, nausea and vomiting. Computerized tomography (CT) of the abdomen demonstrated small bowel obstruction ( $\triangleright$ Fig. 1)

Conservative management with bowel rest, nasogastric suction, and intravenous fluids failed to resolve symptoms. Small bowel obstruction was presumed due to tumor burden. After detailed interdisciplinary discussions with the patient, it was decided to pursue endoscopic guided decompression.

The patient was placed supine on a fluoroscopy table and sigmoidoscopy performed to remove stool and introduce water to promote acoustic coupling. A GF-UCT180 curved linear array echoendoscope (Olympus, Tokyo, Japan) was advanced into the sigmoid colon identifying multiple dilated bowel loops. The bowel was punctured with a 19-gauge EZ Shot 3 needle (Olym- 


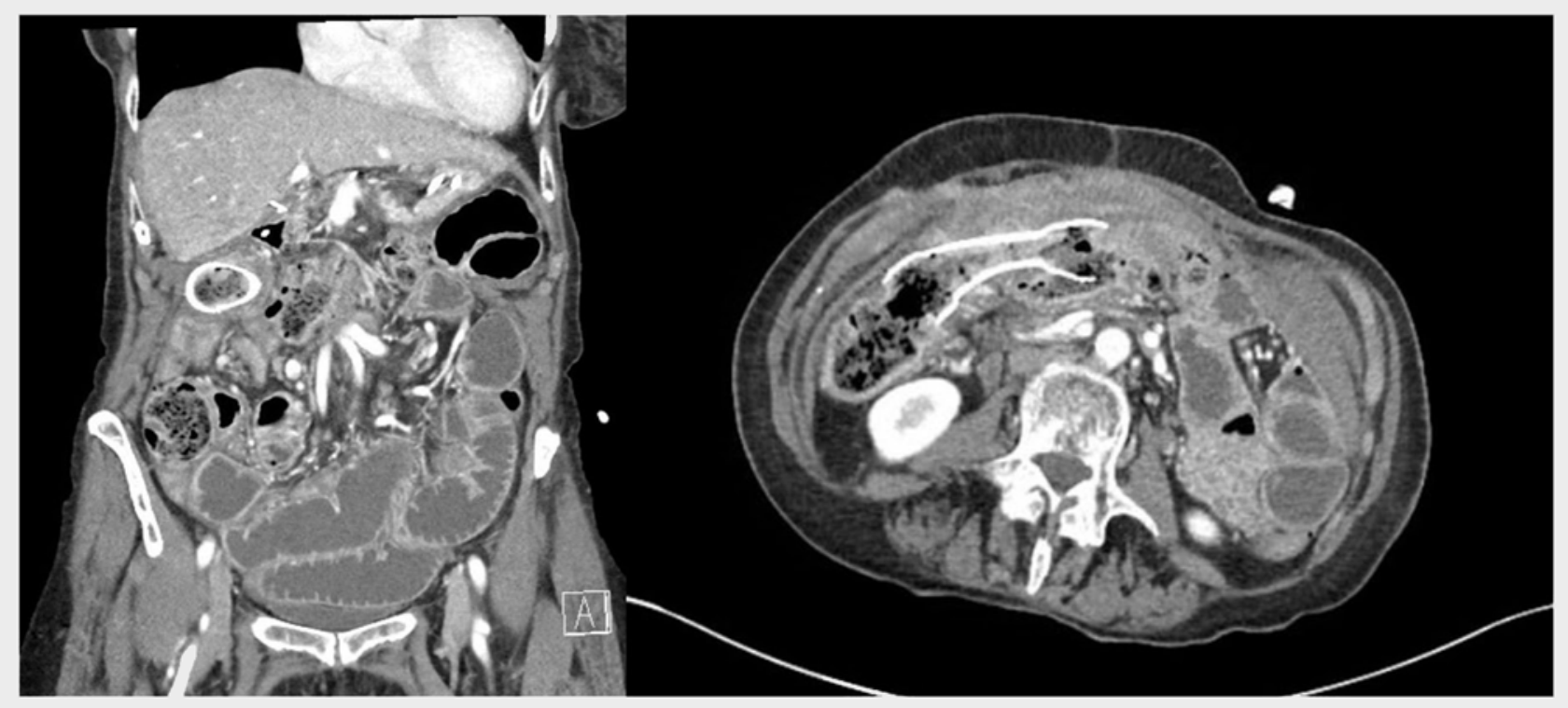

- Fig. 1 Dilated loops of small bowel (left) and previously placed transverse colonic stent (right).
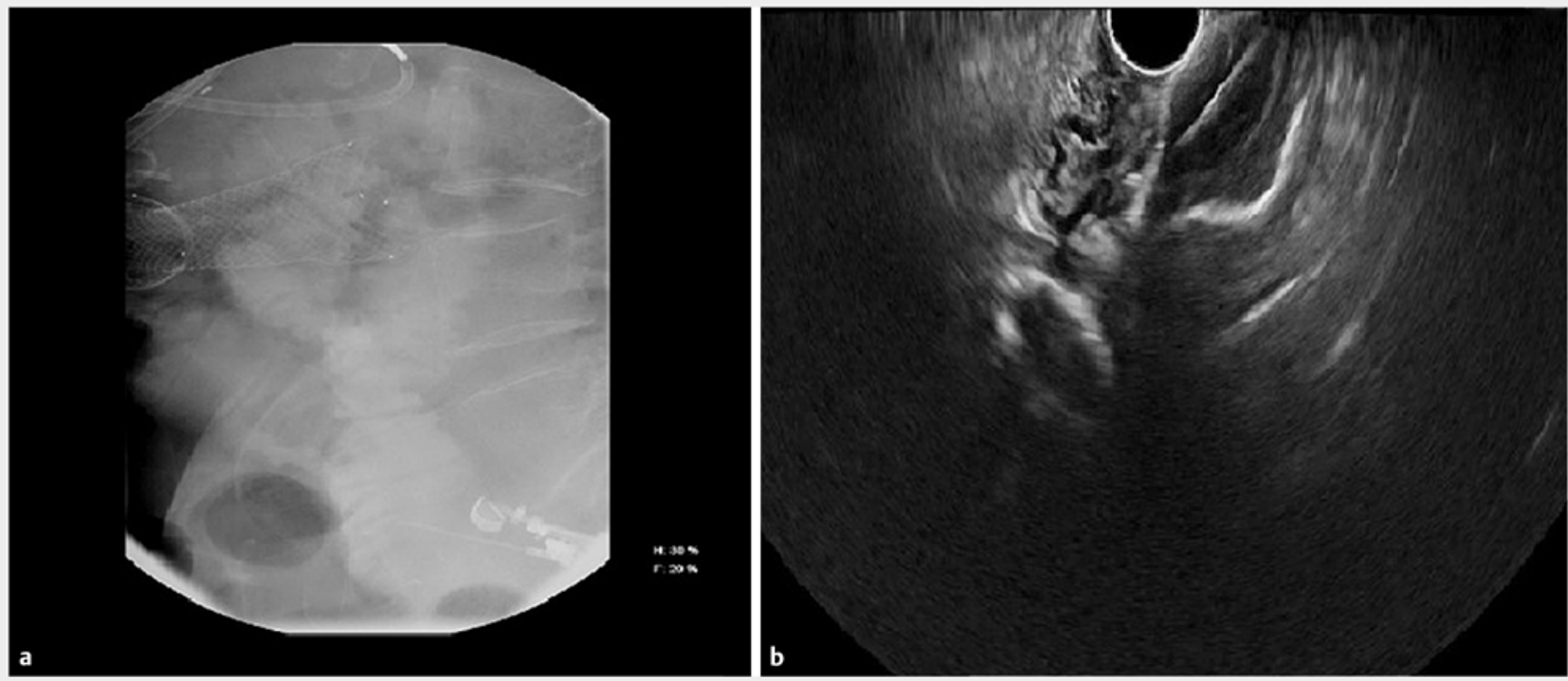

- Fig. 2 a Fluoroscopic image depicting small bowel puncture using the Olympus needle. b Endoscopic ultrasound image demonstrating the AXIOS stent.

pus, Tokyo Japan) and contrast was injected delineating the local small bowel anatomy ( $\mathbf{F i g . 2 a ) . ~ A ~ 0 . 0 2 5 " ~ V i s i G l i d e ~ g u i d e - ~}$ wire (Olympus, Tokyo, Japan) was advanced into the small bowel and the needle exchanged for a $15 \mathrm{~mm} \times 10 \mathrm{~mm}$ AXIOS stent and electrocautery-enhanced delivery system (Boston Scientific, Marlborough, Massachusetts, United States) which was deployed using endoscopic ultrasound (EUS) and fluoroscopic guidance ( $>$ Fig. $\mathbf{2 b}$ ).

The linear echoendoscope was withdrawn, and a sigmoidoscope was used to inspect the stent and visualize the small bowel mucosa through the stent ( $\triangleright$ Fig. 3 ).
Repeat CT scan with oral contrast demonstrated decompression of the small bowel and patency of the enterocolostomy stent ( $>$ Fig. 4 ).

The patient improved symptomatically after the procedure and tube feeding was resumed within 24 hours of the procedure. Antibiotics were continued for 5 days post-procedure. No immediate postoperative AEs were noted with our patient. The patient was subsequently discharged and followed up as an outpatient. After months of progressively worsening metastatic cancer and decline in functional status, the patient opted 


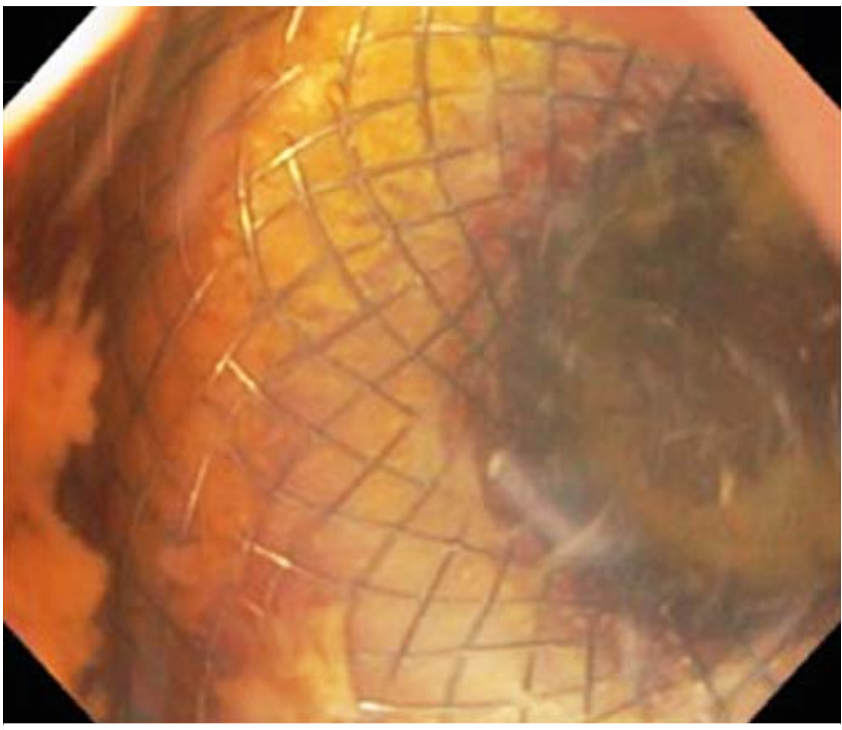

- Fig. 3 Endoscopic image showing the small bowel mucosa through the AXIOS stent.

for palliative care. At this point discussions are underway regarding hospice care.

\section{Discussion}

Three percent to $15 \%$ of cancer patients with intraabdominal tumors have malignant bowel obstruction [2]. It may be acute or chronic; partial or complete. Typically acute and complete obstructions present with severe symptoms compared to partial and slowly developing ones. Also, bowel obstruction can have varying predominance of symptoms depending on the site of obstruction. Typically, proximal obstruction presents with nausea/vomiting as the predominant complaint [3]. It may lead to dehydration, significant electrolyte imbalance, toxemia (related to decomposition of intestinal contents and bacterial overgrowth), bowel infarction and/or perforation [4]. The initial approach includes bowel rest, intravenous fluids, nasogastric decompression and avoidance of medications that impede bowel motility. If conservative measures fail, surgical intervention is often required. However, surgery is a source of significant morbidity in patients with end-stage diseases/malignancies who already have a low life expectancy.

Our case depicts a novel technique to bypass the mechanically obstructed zone as a palliative approach to malignant bowel obstructions. This approach, as described above, not only minimizes perioperative morbidity, but also allows for early functional return, which is essential for patients with already decreased life expectancy. A major challenge to this procedure is the technical ability to choose the most distal loop of bowel among the multiple dilated loops available, which may be complicated by anatomical restraints, and the ability to successfully deploy the stent. Such cases can be technically challenging and the failure to successfully complete the procedure may lead to an iatrogenic perforation requiring emergent surgical intervention and laparotomy.

\section{Conclusion}

EUS use to deploy an AXIOS stent, creating an enterocolostomy resolving malignant bowel obstruction, was successful in the case presented here. Further prospective studies of this procedure will help to better understand the long-term and shortterm effects on morbidity and mortality.

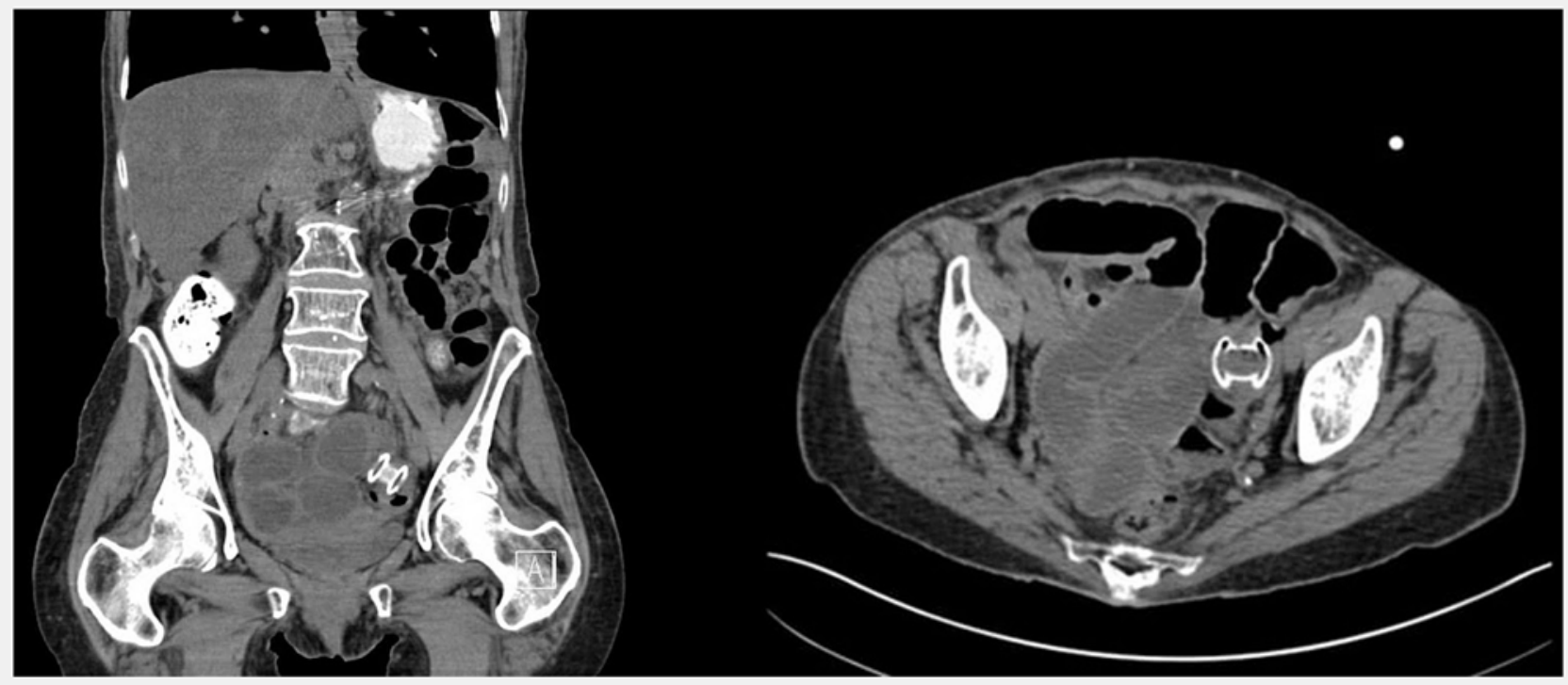

- Fig. 4 Successful placement of the AXIOS stent, coronal section (left) and transverse section (right). 


\section{Competing interests}

None

\section{References}

[1] Paul Olson T], Pinkerton C, Brasel KJ et al. Palliative surgery for malignant bowel obstruction from carcinomatosis: a systematic review.

JAMA Surgery 2014; 149: $383-392$
[2] Tuca A, Guell E, Martinez-Losada E et al. Malignant bowel obstruction in advanced cancer patients: epidemiology, management, and factors influencing spontaneous resolution. Cancer Man Res 2012; 4: $159-169$

[3] Cheadle WG, Garr EE, Richardson JD. The importance of early diagnosis of small bowel obstruction. Am Surg 1988; 54: 565 - 569

[4] Wright HK, O'Brien JJ, Tilson MD. Water absorption in experimental closed segment obstruction of the ileum in man. Am J Surg 1971; 121: $96-99$ 